\title{
Paesaggi contaminati da decontaminare. Qualche riflessione sui luoghi della memoria
}

\author{
di Alberto Cavaglion
}

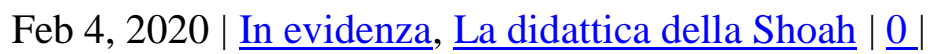

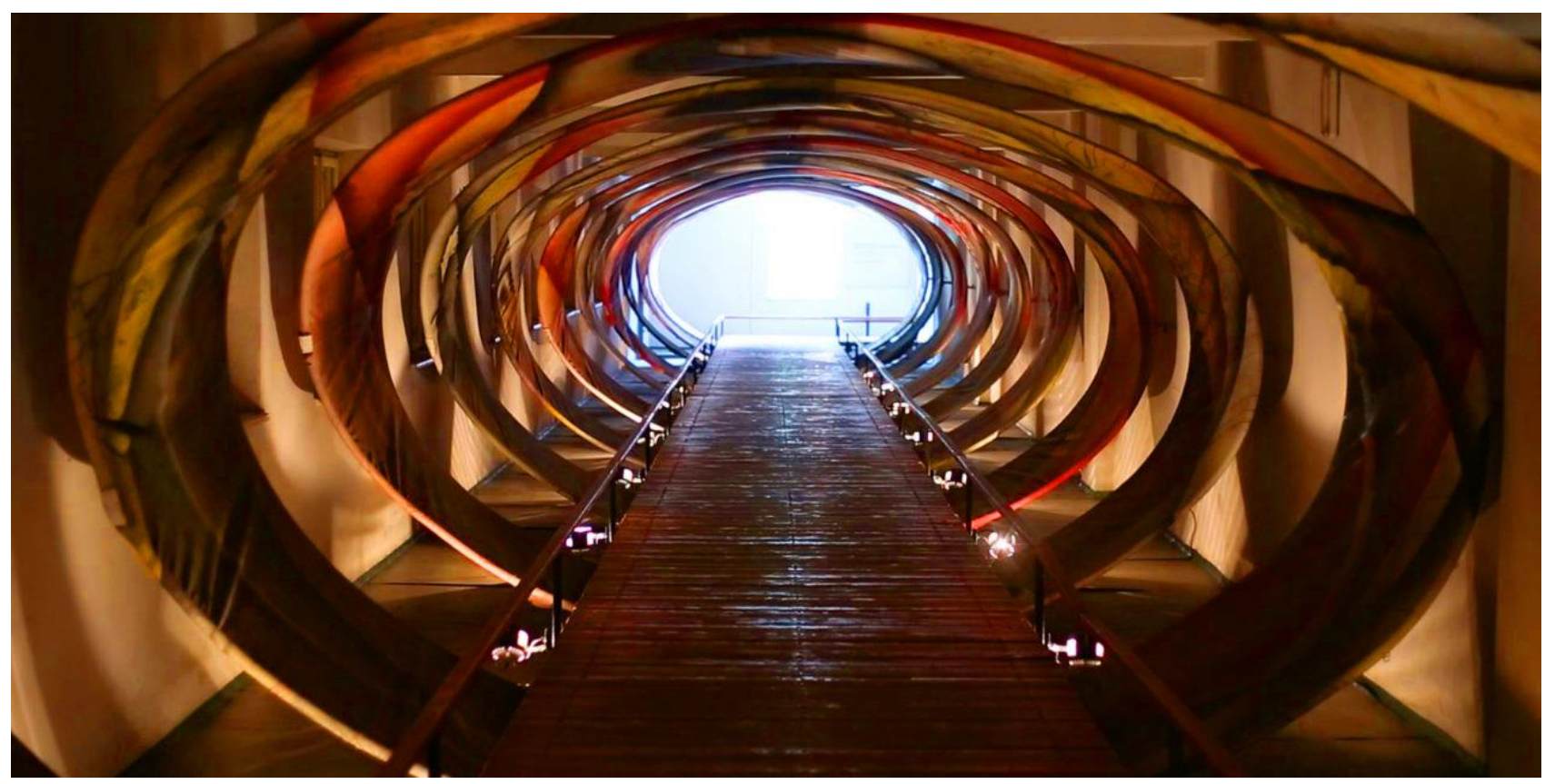

Il memoriale della deportazione italiana, attualmente a Firenze.

Foto di Silvana maja - Own work, CC BY-SA 4.0, Link

\begin{abstract}
La lezione affronta il tema del luogo di memoria prendendo spunto dall'opera di un grande architetto, Gilles Clément, e dal suo Manifesto per il terzo paesaggio. Il primo paesaggio è quello della natura idilliaca, il secondo della natura modificata dall'uomo, il terzo quello della rappresentazione artistica. Si può ipotizzare l'esistenza di un "quarto paesaggio"? Il paesaggio della memoria? Il paesaggio "contaminato" descritto da Martin Pollack può venire in soccorso. La lezione, infine, suggerisce alcuni percorsi bibliografici tratti dalla letteratura (in primo luogo Romain Gary) utili ad aprire nuove prospettive per la salvaguardia dei luoghi della memoria.
\end{abstract}

\section{Premessa}

La discussione sul futuro della memoria, dei memoriali, dei musei del fascismo, delle buone pratiche scolastiche sulla Shoah e il Giorno della Memoria mi sembra sia a un punto morto. Per ripartire occorre rivedere molte delle nostre certezze e progettare con più fantasia il futuro. Quanto è emerso dalla discussione sul Museo del fascismo a Predappio ha messo a nudo una situazione di stallo, ma anche il logorio di vecchi schemi. L'attuale contesto politico rende il quadro più complicato: difficile immaginare se quel Museo potrà essere inaugurato, ma le divisioni che il progetto ha suscitato tra storici e commentatori bastano a mostrare il segno tangibile di uno stato di crisi su cui vale la pena tornare. La categoria di «luogo della memoria» meglio di ogni altra si presta a questo ripensamento. 
Nonostante la lezione dei fatti, dispiace che un sano esercizio di autocritica tardi a diffondersi tra noi che della vecchia stagione siamo stati, chi più chi meno, i protagonisti. Parto, facendole mie, dalle amare riflessioni che Valentina Pisanty ha presentato in queste stesse giornate di Assisi:

Due fatti sono sotto gli occhi di tutti: 1) Negli ultimi vent'anni la Shoah è stata oggetto di intense e capillari attività commemorative in tutto il mondo occidentale; 2) Negli ultimi vent'anni il razzismo e l'intolleranza sono aumentati a dismisura proprio nei paesi in cui le politiche della memoria sono state implementate con maggior vigore. Sono fatti irrelati, due serie storiche indipendenti, oppure un collegamento c'è, ed è compito di una società desiderosa di contrastare l'attuale ondata xenofoba interrogarsi sulle ragioni di questa contraddizione? La constatazione da cui trae avvio il mio intervento è il fallimento delle politiche della memoria, fondate sull'equazione semplicistica Per Non Dimenticare = Mai Più. La domanda è se tale insuccesso sia accidentale (la xenofobia cresce nonostante le politiche della memoria), o se non sia già insito nelle premesse (per come sono state impostate, quelle politiche non potevano che contribuire agli esiti che hanno prodotto). L'obiettivo è predisporsi a combattere la discriminazione in modo efficace e incisivo, che vuol dire anche onesto, consapevole e, ove necessario, autocritico[1].

La categoria di «luogo della memoria» più di altre è entrata in crisi, anche per la sua polivalenza, dal momento che lambisce non una sola sponda: la museologia, le politiche ambientali, le architetture conservative, il turismo scolastico o quello generalista delle vacanze intelligenti. Lo stare insieme, in piccoli gruppi o nei grandi progetti collettivi dei Treni della Memoria ha portato con sé la semplificazione. Non è tanto una questione di contenuti, quanto una carenza di immaginazione, di scenografie, di progettualità creativa. «Comprendere» un luogo flagellato dalla violenza richiede l'intervento di nuovi strumenti, non necessariamente quelli delle moderne tecnologie digitali, che offrono un alibi per rendere più confortevole ciò che per sua natura è afflittivo.

Era stato Pierre Nora a formulare, per primo, la definizione di «lieu de mémoire», una unità d'ordine materiale o ideale, che avrebbe dovuto rendere visibile ciò che non lo era, unendo la storia e la geografia. Nella pratica scolastica, specialmente nelle politiche dei viaggi, con il passare degli anni, come sempre accade quando una idea nuova diventa routine, luogo di memoria è diventato tutto e il contrario di tutto. Un libro, un monumento, una lapide, il luogo di un eccidio, di una battaglia, un personaggio storico, una pietra d'inciampo, tutto è trasformabile in luogo della memoria. Molti luoghi stanno per diventare incroci digitali affollati, centri dell'ipertecnologia, altri sono sul punto di ritornare a essere terra dell'abbandono. Non ancora paesaggi di rovine, ma cristallizzati su se stessi, privi di un domani a fianco di altri spazi postmoderni, tutto immagini in touchscreen e video in loop.

La situazione è confermata da un esempio recente, che non nasce dal discorso su qualcosa che ancora non c'è e forse mai ci sarà (come Predappio), ma da qualcosa che c'era e rischia di non essere più. Mi riferisco alla vicissitudine travagliata del memoriale italiano di Auschwitz, sradicato dal luogo per il quale era stato progettato e trasferito in un non-luogo della Memoria della deportazione. Sia chiaro, a scanso di equivoci: l'operazione del trasferimento da Auschwitz a Firenze è lodevolissima. Abbiamo con ogni probabilità salvato un capolavoro architettonico dal degrado totale o dalla sua demolizione, una demolizione caldeggiata da taluni, fra l'altro nel momento in cui lo scempio stava per essere attuato. Tuttavia, per carenza progettuale, il caso del memoriale rischia di generare due guai anziché uno. Da un lato il rischio che a Firenze, in assenza di un progetto chiaro, ciò che è stato con tanta cura restaurato vada a infoltire la già ricca galleria degli involucri vuoti. Dall'altro lato, il pericolo assai più grave è che lo spazio dedicato ai deportati italiani ad Auschwitz sia destinato chissà per quanto tempo a rimanere vuoto. Vorrei sbagliarmi, ma non ho letto da nessuna parte che si sia levata una voce a spiegarci che cosa intende fare lo Stato 
italiano in quello spazio rimasto vuoto. Il lettore non si aspetti da queste pagine progetti di buone pratiche da attuare subito per salvaguardare luoghi come Marzabotto, Stazzema, Fossoli, Risiera, Fosse Ardeatine, Ferramonti, lo stesso Portico d'Ottavio a Roma. Le considerazioni che seguono si limitano a un paio di proposte, vaghe, in prevalenza libresche, dunque ancora molto distanti dalla attuazione di un progetto. Le buone idee nascono sempre da buoni libri.

Queste pagine muovono da una convinzione consolidatasi nel corso degli anni. Le traversie che la memoria va attraversando penso vadano trasformate in opportunità, a un patto: che tra gli addetti ai lavori, ora lacerati in fazioni litigiose, si sottoscriva un armistizio e si stabilisca una pausa di riflessione. Una pausa che non potrà che essere lunga. Non vedo altre vie di uscita, se si vuole arrivare a progetti realizzabili e a pratiche condivise. Se dipendesse da me, durante questa pausa, invece di lanciare appelli astratti in difesa del tema di Storia nell'esame di Stato prenderei drastiche decisioni: fermerei i Treni della Memoria fino a data da stabilirsi, sospenderei i bandi di concorso per nuovi Musei, indirizzerei le risorse (poche) disponibili, bonus, progetti europei, fondi di incentivazione, a finanziare le biblioteche scolastiche, convogliando lì i docenti «potenziatori». Nulla si è in grado di potenziare in assenza di buoni libri e di spazi accoglienti negli istituti scolastici dove i ragazzi potranno leggerli.

\section{Il quarto paesaggio}

Manifesto del Terzo paesaggio è il titolo di un libro famoso di uno tra i più noti paesaggisti europei[2]. Con questa espressione, Gilles Clément indica qualche cosa che potrebbe intanto aiutarci non a riempire di figure il paesaggio, ma almeno a delimitarne i confini. Il primo paesaggio è quello della natura incontaminata, non toccata dall'intervento dell'uomo. Il secondo è il paesaggio delle arti figurative, della pittura, della poesia, della letteratura. Con «terzo paesaggio» Clément intende $\mathrm{i}$ «luoghi abbandonati dall'uomo» $\mathrm{i}$ parchi e le riserve naturali, le grandi aree disabitate del pianeta, ma anche spazi più piccoli e diffusi, quasi invisibili come le aree industriali dismesse dove crescono rovi e sterpaglie, le erbacce al centro di un'aiuola spartitraffico. Tali sono stati per lunghi anni i luoghi abbandonati dove si è commesso un crimine, un eccidio, una strage, spazi nascosti dove innocenti sono stati reclusi per via dei loro orientamenti politici o per la loro diversità. Questa la ragione che ci induce a immaginare per loro la definizione di «quarto paesaggio».

La connessione tra il terzo paesaggio di Clément e il nostro, per ora ipotetico, quarto paesaggio rappresentato da una memoria storica che si sedimenta nel tempo e raccoglie in comunità gli individui, non è una forzatura, se si pensa che elementi di un quarto paesaggio nelle nostre città sono già visibili nelle aree su cui Clément concentra il suo sguardo, per esempio gli altarini che i ragazzi costruiscono per i coetanei morti schiantati per le strade. Il tempo e le norme sul decoro urbano tendono a cancellarli: finché resta una «comunità di memoria» la necessità del ricordo si conserva, ma come evitare che anche la riflessione sul quarto paesaggio si trasformi in una prescrizione di memoria? Questo il punto cruciale che qui si può soltanto formulare, lasciando a una futura discussione le risposte utili per risolvere un problema complicato. Per molto tempo non solo i luoghi della deportazione e dell'internamento sono stati parte integrante del quarto paesaggio, ma rientrano in questa categoria anche gli spazi più ristretti e anonimi dove si è compiuta una strage, una fucilazione, una tortura. Per molto tempo sono stati paesaggi quasi invisibili. Penso, in Italia, ai decenni dell'abbandono, che hanno rischiato di far cadere nell'oblio i campi di Fossoli o di Ferramonti. Luoghi «minori» come Bolzano o Borgo S. Dalmazzo sono stati così a lungo trascurati da non rendere visibile oggi nessuna traccia del drammatico passaggio, in quelle stanze, dei perseguitati. 
Sono spazi diversi per forma, dimensione e statuto, accomunati dall'assenza di ogni attività umana, ma che presi nel loro insieme sono fondamentali per la conservazione della diversità. Biologica, nel caso studiato da Clément. Storica, nel caso che ci appassiona. Il brillante architetto gioca con le metafore della storia francese: «Terzo paesaggio» rinvia a «Terzo stato» e alle battaglie contro nobiltà e aristocrazia della Rivoluzione: «Cos’è il Terzo stato? - Tutto. Cosa ha fatto finora? Niente. Cosa aspira a diventare il Terzo paesaggio? - Qualcosa»[3]. Noi potremmo seguire le sue orme e porre le stesse domande per il quarto paesaggio, il paesaggio della Memoria. «Cosa aspira a diventare? - Qualcosa». Se nel terzo paesaggio troviamo luoghi in cui l'assenza dell'attività umana ha generato un rifugio per la conservazione della diversità biologica, nel quarto troveremo luoghi in cui la presenza di una politica umana di annientamento ha alterato il paesaggio in modo non facilmente leggibile. Ciò che è «incolto» o ciò che definiamo «erbaccia» diventa qui luogo ed elemento privilegiato del cambiamento ecologico.

Nel quarto paesaggio che proviamo a immaginare, quello della Memoria, troviamo luoghi che conservano la traccia di una condizione estrema di sofferenza, luoghi che richiedono il permanere di una qualche orma della discriminazione politica o razziale. Clément dice che nel terzo paesaggio è centrale l'approccio ecologico che contraddistingue il rapporto uomo/natura e ci invita a percepire la terra come entità viva, come un grande giardino in cui tutti i frammenti di paesaggio ignorati ci offrono opportunità di rigenerazione. Lo stesso dovrebbe valere per il «quarto paesaggio», il paesaggio della memoria, che non potrà mai essere il paesaggio montuoso e interrogativo di Mann o di Musil o il parco divertimenti illuminato di Disney, ma dovrebbe assomigliare forse di più al ciglio della strada, ai bordi dei campi, quelli dove cresce un'erba senza nome, dove la mano restaurativa dell'uomo si ferma. Non è l'infinito, né il finito: è l'indefinito. L'indecisione su quale potrebbe essere il destino del giardiniere del «quarto paesaggio» è il suo rapporto con la cultura degli ambientalisti: la relazione tra letteratura ed ecologia potrebbe guidarci in una direzione innovativa[4].

Clément ci spiega che l'orlo dei campi, una torbiera o un piccolo orto non più coltivato (viene in mente il rivo che scorre accanto alla baracche di Fossoli e ne delimita i confini), un piazzale invaso dalle erbacce o il margine di un'area industriale, laddove non ci sia (o non ci sia più) l'intervento dell'uomo, sono "residui" dove può trovare rifugio la diversità virtuosa, ciò che per noi dovrebbe diventare la scintilla che aiuta a spiegare la diversità di questo luogo di memorie storiche da un altro che ne è privo. L'abbandono, l'isolamento, la solitudine, la riduzione dell'uomo a cosa sono gli elementi caratteristici della prigionia e della deportazione. I residui derivanti dall'organizzazione del territorio (confini dei campi là dove c'erano fili spinati, bordi delle strade là dove si fermavano gli automezzi, i camion che portavano i prigionieri verso la stazione ferroviaria) dovrebbero costituire la materia prima. In uno spazio rurale maggiore è la quantità di residui reperibili. Penso a due luoghi immersi nello spazio rurale come Fossoli di Carpi e Ferramonti, che si dovrebbero prestare, nell'ottica di Clément, non a un freddo restauro conservativo, ma alla creazione di un «quarto paesaggio». In uno spazio urbano, come il centro di Roma dove si è compiuta la tragedia del 16 ottobre 1943, la quantità di residui è minore, anzi si riduce a uno, per altro imponente, come il residuo della romanità imperiale, paesaggio di rovine per antonomasia, ma sono frequenti e visibili i residui naturali in periferia come nel caso delle Fosse Ardeatine. Se minore è l'intervento conservativo, i residui derivanti dall'abbandono evolvono naturalmente verso un paesaggio secondario, che a sua volta sarà interessato da trasformazioni, spiega Clément, fino al raggiungimento di un equilibrio. Nel suo manifesto per il Terzo paesaggio, lo studioso francese invita la società a porsi in due modi diversi verso luoghi come questi: considerarli luoghi naturali da proteggere, assumersi la responsabilità della loro cura e sorveglianza. $\mathrm{Ci}$ sono dunque molti insegnamenti che gli storici della memoria in Italia e anche i docenti possono imparare dai lavori di Clément. Mi rendo conto che il mio Manifesto del quarto paesaggio, così come l'ho formulato, è privo di nomi, di volti, di storie di vita vissuta. Per adesso ci si può limitare - ed è già molto - a 
stabilire la cornice naturale di un diverso concetto di lieu de mémoire. A chi avrà voglia di mettere a frutto la pausa di riflessione che prima sollecitavo spetterà il compito di far sì che il quarto paesaggio si trasformi in un paesaggio con figure.

\section{Paesaggi contaminati}

Un recente saggio di uno studioso di ingegno, Martin Pollack, purtroppo non ha avuto in Italia la fortuna che aveva avuto a suo tempo il libro di Pierre Nora[5]. Il saggio di Pollack non ha la stessa potenza narrativa che hanno i libri di Gerges Perec, Safran Foer o Winfried Georg Sebald. Se dovessi dire a un mio studente «leggi qualcosa intorno a queste idee», continuerei a suggerirgli $W o$ il ricordo d'infanzia, Ogni cosa è illuminata o Austerlitz. Però anche Pollack può aiutarci. Nel suo libro parla di terreni insanguinati, bloodlands, definizione che riprende da Timothy Snyder[6]. Ci invita a scoprire un interesse nuovo per il rimodellamento del paesaggio dovuto alla guerra:

Vogliamo cercare di scoprire che cosa successe qui settanta, ottanta o addirittura cento anni fa, anche se guardando di sfuggita, quando siamo di passaggio, in un'atmosfera rilassata di vacanza, non percepiamo niente che susciti la nostra diffidenza. Ciò nonostante dobbiamo sempre porci la domanda: il paesaggio ha qualche cosa da nasconderci? È davvero così innocente, idilliaco come sembra? Che cosa troviamo se iniziamo a scavare[7]?

Pollack fa riferimento soprattutto alle stragi dell'Europa centrale, alle fucilazioni di massa e alle fosse comuni in Lettonia, che in Italia conosciamo grazie ai libri, sempre preziosi e utili a scuola di Marina Jarre[8]. Che cosa intende Pollack per «paesaggio contaminato», una categoria che dovremmo fare nostra viene spiegato all'inizio del libro:

Con ciò intendo i paesaggi che furono luoghi di uccisioni di massa, eseguite però di nascosto, al riparo dagli sguardi del mondo, spesso con la massima segretezza. E dopo il massacro i colpevoli compiono tutti gli sforzi immaginabili per cancellarne le tracce. I testimoni scomodi vengono eliminati, le cave in cui sono stati buttati i morti vengono riempite di terra [...][9].

Qui intendo servirmi del libro di Pollack non tanto per il contenuto del libro in sé, ma per quello che non dice e solo lascia intendere. Osservo intanto che Pollack svolge nel suo libro un ragionamento a ben guardare non lontano da quello di Clément e, indirettamente, ci offre una metafora di partenza molto interessante, «paesaggi contaminati». Con questa espressione Pollack spiega l'incursione della storia, della malvagità umana nel paesaggio naturale: il primo paesaggio cessa di diventare idillico e si trasforma in un paesaggio malato, simile a quello che è passato attraverso una calamità naturale o ecologica. Il ruolo che ha avuto l'uomo nella contaminazione dell'idillio della Natura è dunque duplice: si distrugge per fini economici o per mera brutalità.

Il visitatore, il turista, lo studente, davanti alle baracche di Fossoli o alle cascine bruciate di Stazzema o Boves, dovrebbe essere invitato a immaginare come si può fare per de-contaminarlo, quel paesaggio, adattando le categorie di Clément o le riflessioni di Pollack. Al suo ingresso in un luogo deturpato dall'uomo al punto da renderne irrespirabile l'aria, il visitatore, prima di essere informato sulla storia specifica di quel luogo, dovrebbe essere così avvertito: «Stai per entrare all'interno di un paesaggio contaminato, anche tu devi contribuire a pensare come e che cosa si dovrebbe fare per purificarlo». Clément e Pollack ci possono servire, ma l'applicabilità delle loro norme non potrà essere automatica. Per decontaminare un paesaggio è necessario, come prima cosa, sapere che è contaminato. Altro dettaglio non trascurabile: Pollack parla di paesaggi che scopriamo 
essere contaminati, ma che nessuno vorrebbe si sapesse. Noi dobbiamo invece immaginare un futuro per «luoghi della memoria» istituzionalizzati. Non è propriamente la stessa finalità.

Come si presentano questi luoghi oggi? C'è qualcosa che li distingue dai luoghi innocenti, dove il paesaggio non ha nulla da nascondere? Per l'Italia il discorso sul nascondimento tocca soprattutto un luogo della memoria come le Fosse Ardeatine, la cava nel terreno dove furono gettati i morti, le foibe stesse. Pollack si spinge più in là nell'osservazione dei paesaggi contaminati, si sofferma in modo toccante sui dettagli, così come dovremmo fare noi quando ci aggiriamo nei dintorni di ciò che resta delle baracche di Fossoli, degli arbusti che sono cresciuti fino ad avvolgere i brandelli di muro:

Quando raccolsi qualche foglia, vidi che presentavano tutte delle strane macchie nere, che interpretai come un'allusione alla cupezza della località verso cui ero diretto. Come se la natura avesse voluto annunciare la vicinanza del luogo delle uccisioni. Solo molto tempo dopo venni a sapere che il disegno nero della foglia su cui mi ero fermato a pensare era stato causato da una circostanza banalissima. Erano i sintomi di una malattia fungina molto diffusa in Europa, Rhytisma acerinum, che colpisce diversi tipi di acero. Si tratta di un fungo parassita che forma sulle foglie fitti intrecci che assomigliano a motivi neri. Come se fossero a lutto. Allora avevo preso qualcuna delle foglie macchiate di nero e le avevo conservate a casa finché non divennero fragili e non mi si sfaldavano tra le mani[10].

Pollack intervista anche la gente del luogo, come dovremmo fare noi chiedendoci se la natura ha ripreso a fare il suo corso, se è tornato abituale risiedere in un paesaggio contaminato e quanto tempo è necessario perché la decontaminazione avvenga. Dice il contadino-testimone, in tono di rimprovero: «Qui, dove adesso ci sono i pali di legno, niente è mai venuto su bene, non importa cosa volessimo coltivare, rape, cavoli, kartoshka, grano saraceno»[11]. Viene in mente la lezione del Treblinka di Vassilij Grossman, dove è il paesaggio a ribellarsi, almeno secondo l'autore: hai voglia a livellare e fare orti, ma dalla sabbia escono di continuo i resti che accusano[12].

Il caso-Fossoli è quello che più agevolmente si presta a essere inserito in un discorso come questo, perché le sue stesse stratificazioni e la sua storia sono un processo di lenta de-contaminazione. Negli anni compresi tra il 1947 e 1970, la sua funzione è mutata radicalmente, anche se in stretta relazione con la pesante eredità che la guerra ha lasciato. Da luogo di detenzione e coercizione ha dovuto rispondere a esigenze abitative che comportano la riorganizzazione degli spazi e interventi sulle baracche per adattarli alla vita in civile. Il primo capitolo si apre con il trasferimento nel campo della Comunità dell'Opera dei Piccoli apostoli, fondata da don Zeno Saltini. Si tratta di ragazzi difficili o abbandonati che don Zeno ha raccolto fin dagli anni Trenta il cui numero è cresciuto vertiginosamente a causa della guerra. Sarà opera loro togliere il filo spinato, abbattere i muri, dissodare e addomesticare l'area per renderla un luogo dove ricostruire una vita comune dopo la tragedia della guerra. I progetti per la definizione degli spazi comuni, per le trasformazioni delle baracche in accoglienti unità abitative, per la sistemazione del verde e degli orti, ribaltano in modo evidente la funzione che durante la guerra ha avuto Fossoli e gli assegnano il senso completamente opposto di fratellanza. Nel breve tempo che la comunità di don Zeno resta a Fossoli, dal 1947 al 1952, gli interventi realizzati sono parziali rispetto alla progettazione, ma da lì potranno partire le famiglie italiane che dal 1954 arriveranno dall'Istria dopo la firma del Memorandum di Londra che assegna in modo definitivo le terre dove abitavano alla Repubblica Iugoslava. Lo spazio in cui le famiglie giuliane entrano a vivere dal 1954 ha ormai perso gli elementi più evidenti della reclusione, anche se restano identificabili i segni del campo di concentramento nella uniformità delle baracche e nell'impianto a "caserma" ancora molto evidente. 
Ciò che noi oggi vediamo attraversando la parte superstite del campo di Fossoli è dunque frutto di una stratificazione che si è depositata via via sulle strutture originarie nel corso della sua lunga storia. D'altra parte, la materialità del luogo conserva diverse tracce da cui possiamo partire per indagare le diverse storie, di guerra come di ricostruzione, che si incrociano anche quando ci muoviamo fra le mura della Risiera di San Sabba.

Quello che Pollack non dice, e invece dovremo immaginare noi, riguarda il processo successivo, della de-contaminazione. Una volta avvertito lo studente che si appresta a un luogo malato, resta la parte più gravosa per chi deve insegnare tecniche di superamento. Meglio forse che resti così com'è, senza interventi, piuttosto che retrocederlo da quarto a secondo paesaggio, dove la mano trasformatrice dell'uomo sulla Natura risulterebbe annientatrice. Nella linea dei tre (o quattro) paesaggi, che poi è la linea del tempo e della storia, non si fanno salti, ma non si torna nemmeno indietro. A noi spetta il compito, che soggettivamente è anche un dovere, di suggerire a chi verrà dopo di noi un percorse di guarigione, di rigenerazione, in una parola di speranza.

\section{Aquiloni}

Un passaggio obbligato nella didattica della Shoah, in particolare nella presentazione dei luoghi della memoria, riguarda il nesso paura-speranza. In molti dei percorsi scolastici che mi è capitato di osservare, la pedagogia della violenza, l'abuso della memoria orrorosa hanno prevalso sulla pedagogia della de-contaminazione. Se adesso vogliamo seguire le orme di Pollack, non possiamo fare a meno di indicare un percorso che rappresenti la rivincita della civiltà sulla barbarie e indichi una linea che dalla contaminazione orienti lo studente verso nuove competenze: cogliere il tragico della storia e allo stesso tempo coltivare la speranza, sulla base di sentimenti di solidarietà, comunanza, comprensione umana. La vita insomma, con la storia e, soprattutto, la letteratura.

Pier Vincenzo Mengaldo ha trovato la formula giusta quando ha parlato della «vendetta del racconto» ovvero della funzione riparatrice della letteratura[13]. Dove in passato abbiamo sbagliato? Puntando su testi o scrittori che hanno fondato il racconto sul solo dovere della memoria abbiamo trascurato lo statuto della letteratura, un suo punto essenziale: il valore rasserenante della finzione letteraria. Forse, puntare sul dovere di ricordare, rappresenta una parte dei nostri errori. Poiché la critica deve sempre essere rivolta anche contro se stessi, riconosco che molti dei percorsi suggeriti ai miei studenti intorno alla affascinante figura di Georges Perec e del suo «souvenir d'enfance» partivano da un errore di fondo: ritenere fecondo il gioco virtuosistico che Perec fa della frase «mi ricordo», del suo essere un ragazzo dotato di una straordinaria memoria, una specie di imperativo categorico che non transita dalla pagina dei suoi libri alla quotidianità di uno studente odierno di scuole superiori. In modo analogo, si è iniziato e si è continuato a leggere lo stesso Levi nel corso degli anni. Anche sull'uso di Se questo è un uomo e dei Sommersi e i salvati, sul consolidarsi di un ipse dixit, vi sarebbe da dire, sull'eccesso prescrittivo di molte letture scolastiche di Primo Levi.

L'alternativa forse va cercata nelle forme che la letteratura ha saputo creare in dialogo con la Natura, quello che Clément chiamerebbe il secondo paesaggio, il paesaggio dell'arte.

Per bonificare i luoghi contaminati e ricostruire, supponiamo, l'idillio del paesaggio agreste carpigiano dentro cui si trovano immerse le baracche di Fossoli, i luoghi dell'immaginazione che la storiografia economica può fornire diventano utili parametri di confronto. Certo, sempre si tratta di suggerimenti bibliografici, troppo poco per la edificazione di un luogo. Penso a un classico libro di storia economica, la storia del paesaggio di Emilio Sereni, che nella sua prima edizione reca uno 
straordinario apparato iconografico dove la pianura padana, che ha visto la tragedia dei fratelli Cervi, la strage di Marzabotto, accolto Primo Levi o i bambini di Nonantola, ci viene restituita nella sua naturale bellezza, con le planimetrie antiche, le tavole degli studiosi di agronomia e, soprattutto, le meraviglie dell'arte figurativa del Medioevo e del Rinascimento, cancellando magari la sofferenza di chi quel paesaggio doveva lavorare come servo, come contadino[14].

Mi rendo conto di quanto sia vago il mio «manifesto per il quarto paesaggio». Mi accorgo, mentre scrivo, di riuscire a fornire solo un percorso libresco e, nonostante la critica iniziale, non riesco a svincolarmi dall'impostazione di Nora: memorie collettive, geografia e storia, sotto l'ala dello Stato e della memoria di Stato. Il mio discorso resta principalmente di una istituzione pubblica e di rituali collettivi (musei pubblici, viaggi sui luoghi finanziati da enti pubblici, posa di lapidi o di pietre d'inciampo), ma non trascurerei esplorazioni nuove al fine di costruire quarti paesaggi collocabili al di fuori di questo ambito istituzionale, magari molto piccoli, ma capaci di stimolare la competizione con gli enti pubblici.

Ciascuno di noi sogna di fuggire altrove. E d'altra parte fin dall'inizio ho avvertito che ci dovremmo concedere una lunga pausa di riflessione. Come riempire l'attesa se non con qualche buona lettura? Il presente in cui siamo immersi più che mai induce alla ricerca di un museo che non c'è, di un memoriale che vorremmo costruito secondo i nostri sogni, per trasmettere un segnale di speranza. Siccome, come dicevo, la pausa sarà lunga e anche quando avremo chiara la struttura del quarto paesaggio ci vorrà del tempo per farne un paesaggio con figure, nella attesa, un buon romanzo può sempre essere di sollievo per tutti. Questa la ragione per cui, a corollario della mia modesta proposta, in conclusione, accanto a Pollack, colloco un romanzo non fra i più celebri di Romain Gary, Gli aquiloni[15].

Una dimensione spaziale simmetrica divide i miei due suggerimenti di lettura. Se la terra, secondo Pollack, reca visibili i segni del contagio, nessuno ci dovrebbe vietare, mentre pensiamo, di alzare gli occhi al cielo e «rincorrere l'azzurro» come suggerisce Gary. Non si può fare a meno, visitando un paesaggio contaminato, di ricorrere a un infantile esercizio di sopravvivenza che il grande scrittore francese ci suggerisce: giocare con gli aquiloni è arte delle ingenuità dell'infanzia, «la parte più feconda che la vita ci dà e poi si riprende»[16].

Il libro di Gary si apre con una mossa spiazzante, la notizia di un piccolo museo che «merita una puntatina». In questi anni di generale sconforto, mi è capitato spesso di ritornare a questo libro e di immaginare una puntatina in un Museo che in Italia non c'è:

Oggi il piccolo Museo dedicato alle opere di Ambroise Fleury, a Cléry, rappresenta soltanto un'attrazione turistica minore. Perlopiù $i$ visitatori ci vanno dopo un pranzo al Clos Joli, che tutte le guide di Francia sono unanimi nel celebrare come uno dei luoghi d'eccellenza del paese. Le guide comunque segnalano l'esistenza del museo con la dicitura «merita una puntatina». Nelle sue cinque sale si trova la maggior parte delle opere di mio zio che è sopravvissuta alla guerra, all'occupazione, alle battaglie della Liberazione e a tutti gli accadimenti e a tutti i cedimenti che il nostro popolo ha conosciuto[17].

Ogni volta che leggo notizie di dibattiti sui musei di storia contemporanea, ogni volta che visito un museo o una mostra della deportazione, trovandomi dinanzi a progetti faraonici, ambiziosi mi capita di esortare i miei colleghi a leggere Gli aquiloni: Gary aiuta a sognare a occhi aperti, a fantasticare con l'ingenuità dell'infanzia e predisporsi a inaugurare piccoli musei dove la memoria del luogo sia garantita, da artigiani costruttori di aquiloni come Ambroise Fleury, il protagonista del romanzo: «Bisogna tenerli saldamente», spiega al nipote, «perché tirano e certe volte ti sfuggono di mano, salgono troppo in alto, si mettono a inseguire l'azzurro e non li rivedi più, a men che la gente non te 
li riporti a pezzi». Fleury chiama gnamas i suoi aquiloni: «Aveva scoperto questa parola in un'opera sull'Africa equatoriale, dove, a quanto sembra, definisce tutto ciò che ha un alito di vita, uomini, moscerini, leoni, idee o elefanti»[18]. Di gnamas bisogna farne continuamente di nuovi: Fleury era partito dalla memoria storica della Francia, rabberciava con ago e filo l'effigie di Carlo Martello, Giovanna D'Arco, Rousseau, Napoleone. Con l'inizio della guerra costruisce gnamas con il viso di De Gaulle e Pétain: i tedeschi che occupano il suo piccolo paese in Normandia lo costringono a farli volare bassi, lo perseguitano. Quando Fleury viene a sapere degli arresti del Vel d'Hiv la sua consapevolezza politica cresce, viene a sapere dei bambini confluiti a Chambon-sur-Lignon e assistiti dal pastore André Trocmé. Parte e raggiunge quel luogo di assistenza, continuerà a costruire gnamas per quei bambini, fino a che viene arrestato e deportato ad Auschwitz, ma nemmeno lì si ferma. Bisogna sempre continuare a farne di nuovi. Tra nipote e zio si instaura una competizione sul filo della memoria: il ragazzo è un piccolo Perec, «un piccolo mostro» che ricorda tutto. Ripete a memoria dieci pagine dell'orario ferroviario Chaix: «i Fleury hanno una memoria così buona che qualcuno di loro ne è morto», ma il suo primo maestro e lo zio gli spiegano che una memoria prodigiosa non può essere una dote: «L'intelligenza presuppone il ragionamento. Insisto su questo: il ragionamento». La facilità di tenere tutto a mente, se non è accompagnata da un progresso nella maturità intellettuale, nella ponderazione e nel buon senso è fonte di disastri. Invece, inseguire l'azzurro con il filo dei gnamas «insegna a prendere in giro se stessi e il proprio eccesso di memoria»[19].

Mi rendo conto che si tratta di un sogno a occhi aperti e che non saprei cosa suggerire a uno studio di architetti che volesse costruire, in pratica, un museo dove la storia si insegna come vorrebbe Ambroise Fleury. Non ho soluzioni pratiche da offrire, solo suggerimenti per impiegare bene il tempo lungo che la pausa di riflessione ci potrà offrire. Senza l'assillo di dover riempire musei, senza l'affanno di far partire Treni della Memoria la letteratura può regalarci la speranza. E magari, in tempi brevi, un laboratorio per l'infanzia sulla Shoah costruito attraverso l'artigianato degli aquiloni e il libero gioco della fantasia: non sarebbe una nota stonata, né impresa ardua costruire quegli oggetti volanti con ago e filo per finire poi tutti insieme a rincorrere l'azzurro sui terreni contaminati. Per iniziare bene un cammino, e accontentarsi di poco, in attesa che i tempi migliorino, potrebbe essere un primo segnale incoraggiante, la prova di un'inversione di tendenza.

\section{Note:}

[1] Si veda Valentina Pisanty, Che cosa è andato storto? Le politiche della memoria nell'epoca del post-testimone, Novecento.org, n. 13, febbraio 2020 [http://www.novecento.org/la-didattica-dellashoah/che-cosa-e-andato-storto-le-politiche-della-memoria-nellepoca-del-post-testimone-6297//], url consultata il 4 febbraio 2020

[2] G. Clément, Manifesto del Terzo paesaggio, Quodlibet, Macerata 2004.

[3] Clément, 2004, p. 7.

[4] N. Scaffai, Letteratura e ecologia. Forme e temi di una relazione narrativa, Carocci, Roma 2018.

[5] M. Pollack, Paesaggi contaminati. Per una nuova mappa della memoria in Europa, Keller edizioni, Rovereto 2014. 
[6] T. Snyder, Terre di sangue: l'Europa nella morsa di Hitler e Stalin, Rizzoli, Milano 2011.

[7] Pollack, 2014, p. 69.

[8] M. Jarre, Ritorno in Lettonia, Einaudi, Torino 2003.

[9] Pollack, 2014, pp. 26-27

[10] Pollack, 2014, p. 103.

[11] Pollack, 2014, p. 114

[12] V. Grossman, L'inferno di Treblinka, Adelphi, Milano, 2010.

[13] P.V. Mengaldo, La vendetta è il racconto: testimonianze e riflessioni sulla Shoah, Bollati Boringhieri, Torino 2007.

[14] E. Sereni, Storia del paesaggio agrario italiano, Einaudi, Torino 1962.

[15] R. Gary, Gli aquiloni, Neri Pozza, Vicenza 2017.

[16] Gary, 2017, p. 68.

[17] Gary, 2017, p. 17.

[18] Gary, 2017, p. 9.

[19] Gary, 2017, p. 29. 